\title{
Kronos: a multiwavelength observatory for mapping accretion-driven sources
}

\author{
Bradley M. Peterson ${ }^{* a}$, Ronald S. Polidan ${ }^{* * b}$, and Edward L. Robinson ${ }^{* *} c$ \\ ${ }^{a}$ Department of Astronomy, The Ohio State University; ${ }^{b}$ NASA's Goddard Space Flight Center; \\ 'Department of Astronomy, University of Texas at Austin
}

\begin{abstract}
Kronos is a multiwavelength observatory proposed as a NASA Medium Explorer. Kronos is designed to make use of the natural variability of accreting sources to create microarcsecond-resolution maps of the environments of supermassive black holes in active galaxies and stellar-size black holes in binary systems and to characterize accretion processes in Galactic compact binaries. Kronos will obtain broad energy range spectroscopic data with co-aligned X-ray, ultraviolet, and optical spectrometers. The high-Earth orbit of Kronos enables well-sampled, high time-resolution observations, critical for the innovative and sophisticated methods that are used to understand the accretion flows, mass outflows, jets, and other phenomena found in accreting sources. By utilizing reverberation mapping analysis techniques, Kronos produces advanced high-resolution maps of unprecedented resolution of the extreme environment in the inner cores of active galaxies. Similarly, Doppler tomography and eclipse mapping techniques characterize and map Galactic binary systems, revealing the details of the physics of accretion processes in black hole, neutron star, and white dwarf binary systems. The Kronos instrument complement, sensitivity, and orbital environment make it suitable to aggressively address time variable phenomena in a wide range of astronomical objects from nearby flare stars to distant galaxies.
\end{abstract}

Keywords: Black holes, gravitational accretion, ultraviolet spectroscopy, X-ray spectroscopy, tomography

\section{INTRODUCTION}

Gravitational accretion onto black holes produces a significant fraction ( 10 to $50 \%)$ of the radiation emitted by discrete sources over the history of the Universe and powers some of the most luminous objects known. Thus, understanding accretion processes is as important as understanding how stars shine. The small angular sizes of even the nearest black-hole systems (tens of microarcseconds) necessitate the use of technically challenging nontraditional methodologies, such as those used by the Kronos multiwavelength observatory.

The fundamental goal of the Kronos mission is to understand accretion onto black holes and other compact sources. Kronos will determine what accretion structures and radiation mechanisms are found in nature over the range of well-established black-hole masses $\left(10-10^{10} M_{\odot}\right)$ in Galactic binaries and in active galactic nuclei (AGNs). Kronos will map the environments of black holes and other compact accretion-driven sources to determine how high-energy radiation $(\mathrm{X}$-ray, ultraviolet, and optical) is produced and reprocessed, to determine how the accretion flows are related to outflowing winds, jets, and other features of accreting sources, and to discover why these features appear in some objects but not in others.

Kronos addresses these problems through mature and sophisticated tomographic techniques that yield microarcsecondresolution maps of accretion-driven sources with levels of spatial detail that are generally considered to be unachievable, in the X-ray, ultraviolet, or optical, by any other means within the next 20 years. This is accomplished through proven timingbased methods exploiting the natural variability of accretion-driven sources:

\footnotetext{
-peterson@astronomy.ohio-state.edu; phone 1614 292-7886, fax 1614 292-2928; http://www.astronomy.ohio-state.edu/-kronos/; Department of Astronomy, The Ohio State University, 140 West $18^{\text {th }}$ Avenue, Columbus, OH 43210 , USA.

** Ronald.Polidan@kirtland.af.mil; phone 1 505 853-3870; fax 1 505 846-0959; Space Vehicles Directorate, Kirtland Air Force Base, NM 87117 , USA.

*** elr@astro.as.utexas.edu; phone 1512 471-3401; fax 1 512 471-6016; Department of Astronomy, University of Texas, Austin, TX 78712 , USA.
} 
1. Reverberation mapping ${ }^{2,25,27}$ uses the detailed response of AGN broad emission lines to continuum variations in determining geometry and kinematics of the broad-line region (BLR).

2. Eclipse mapping ${ }^{13}$ uses the progressive occultation of the accretion disk by the cool secondary in high-inclination binaries in mapping the surface brightness distribution of the accretion disk. Kronos will do this during outbursts, thus tracking the evolution of the accretion disk structure.

3. Doppler tomography ${ }^{19}$ maps out accretion-disk structure through line-profile variations.

4. Mulitwavelength monitoring of continuum variations in both blazars (AGNs whose spectra are dominated by jet emission) and non-blazars is used in determining causal relationships between variations at different photon energies, thus identifying physical mechanisms at work.

Like astronomical interferometry, Doppler tomography and reverberation mapping are indirect imaging methods, much like seismology or computed axial tomography (CAT scans). While interferometry relies on source structure remaining constant over the duration of an observation, tomographic techniques exploit the nature variability of accretion-driven sources to probe their structure. However, the methods identified above have reached natural limitations, imposed primarily by the temporal coverage achievable from low-Earth orbit (LEO) or from the ground. Kronos overcomes these limitations by utilizing a high-Earth orbit (HEO) that permits long, uninterrupted observations for time-series analysis. The key element of Kronos is that it is dedicated to long-term monitoring programs. Whereas other missions return snapshots, Kronos returns movies.

The defining features of Kronos are:

1. The Kronos observatory is designed specifically for long-term, high time-resolution observations of accretion-driven sources. The Kronos HEO provides continuous on-source times of up to nearly the orbital period of $\sim 14$ days with only rare interruptions.

2. The Kronos telescopes cover the most important wavelength regimes for accretion-driven sources. The throughput of the X-ray and ultraviolet/optical telescopes ensures that the on-source times are determined by timescales of physical processes, not by photon arrival rates.

3. Kronos is primarily a key-projects mission. The highest science return will be achieved through very long observations of a limited number of carefully chosen representative sources.

4. Kronos is designed for flexibility. Changes in observing schedule are easily implemented. Data are in the form of photon lists or short-integration spectral images, which allow post-facto optimal binning in wavelength and time to achieve different science goals using the same data.

\section{Active Galactic Nuclei}

Approximately 5 to $10 \%$ of all bright galaxies have sub-parsec-scale nuclear activity not obviously attributable to stars. These galaxies have active galactic nuclei that emit copious radiation across the spectrum. In the optical band, the central source is as bright as the host galaxy in lower-luminosity AGNs (Seyfert galaxies) and at least 100 times brighter in high luminosity AGNs (quasars). On the basis of simple arguments ${ }^{26}$, it has been long suspected that AGNs are powered by gravitational accretion onto supermassive black holes, and that other AGN features, such as strong emission lines and relativistic jets, are by-products of this process ${ }^{8}$. There is now strong consensus that supermassive black holes reside in the cores of most if not all galaxies, and the questions about the differences between active and quiescent galaxies are couched in terms of differing accretion rates. It seems likely that the broad emission lines are related to the accretion process, and thus determination of the geometry and kinematics of the BLR assumes central importance in understanding quasar structure and accretion modes.

\subsection{Reverberation mapping}

The essence of the reverberation technique is to measure, in detail, the emission-line response to continuum variations. The time lag between continuum changes and the line response is the light travel time between the continuum source and the responding gas. Because the emission lines are resolved in wavelength, this can be done as a function of Doppler shift. This requires a time series of spectra that cover several prominent $U V$ and optical emission lines, the FUV continuum, and the soft $\mathrm{X}$-ray continuum (which produces the highest ionization species). The primary data product is the emission-line response as a 
function of line-of-sight velocity (or "transfer function"). The transfer functions then are used to generate images of the central region via maximum entropy techniques or by modeling.

Successful reverberation mapping requires a set of high time-resolution, long-duration, moderate wavelength resolution, high signal-to-noise ratio $(S / N)$, homogeneous, multiwavelength spectra. A complete map of the BLR requires several emission lines of different ionization levels and distances from the central source because the BLR has a stratified ionization structure (e.g., highest ionization lines arising closest to the central source). To date, all reverberation-mapping programs have been deficient in one or more of the key qualities listed above. Consequently, the only clear results have been the BLR radius in 36 AGNs, far below the full potential of microarcsecond maps achievable through reverberation techniques.

By design, Kronos avoids all of the deficiencies of previous reverberation monitoring. We have carried out extensive numerical simulations ${ }^{14}$ (see also Horne, these proceedings) demonstrating that, unlike previous experiments, Kronos yields high-fidelity transfer functions, leading to high-fidelity maps of the BLR. Reconstructions with Kronos reverberation data will yield a definitive image of the central regions of 5 to 10 bright AGNs whose variability properties have been wellcharacterized by previous work with an effective resolution 10,000 times more detailed than HST images.

\subsection{Black-Hole Masses}

One of the most important results of reverberation mapping is measurement of central black-hole masses. The BLR radius $R$ can be combined with the emission-line Doppler width $V$ to yield a mass estimate based on the virial theorem, $M=f R V^{2} / G$, where $f$ is a factor of order unity that depends on the geometry and kinematics of the BLR ${ }^{36,15}$. The problem with current reverberation-based virial masses is that, without knowledge of the geometry and kinematics of the BLR, the systematic errors remain unknown. This is a significant issue because, in some cases, the systematic uncertainties can be extremely large, up to factors of several ${ }^{10.16}$. This problem can only be resolved by actually measuring the BLR structure and kinematics by reverberation mapping. This is a major Kronos goal. A detailed map of the BLR in even one AGN would provide a means to assess how large systematic errors can be. Our simulations indicate that 10 to $20 \%$ accuracy in black-hole masses is routinely achievable with Kronos.

The importance of this effort should not be underestimated. Reverberation masses are already providing the comerstone for other methods of AGN black-hole mass estimation ${ }^{17.3635 .23}$, and, therefore, the systematics must be completely understood. This is important not only in the context of the masses already estimated, but also because of the potential of reverberation methods under more extreme conditions. For example, this technique is easily extendable to higher redshift sources, so the evolution of AGN masses and accretion rates over cosmological timescales can be studied. Also, because the reverberation method is based on gas motions on scales that are tiny by galactic standards, it provides a way to measure even the smallest supermassive black holes $\left(<10^{6} M_{\odot}\right)$, which are otherwise undetectable by standard dynamical methods in non-AGNs. Reverberation masses must be understood completely as they will be of tremendous importance in the demographic studies leading to understanding the formation and evolution of black holes and their host galaxies ${ }^{24}$.

\subsection{AGN Continuum Emission}

Continuum emission from AGNs is due to the poorly understood interplay of coupled thermal and non-thermal processes. Through the emission-line reverberation-mapping program, Kronos provides simultaneous data to compare continuum variations in the hard X-ray, soft X-ray, UV, and optical. These data will constrain the temperature structure of the accretion disk and the temporal relationship among the wavebands, and, thus, identify the primary variable energy source and determine the origin of the hard $\mathrm{X}$-rays.

\subsection{Jet Emission in Blazars}

The continuum emission of blazars (BL Lacertae objects and highly polarized quasars), the most violently variable type of $\mathrm{AGN}^{33}$, is dominated by nonthermal radiation from a jet shooting outward at a bulk Lorentz factor of $\sim 10$ and directed close to our line of sight. With such relativistic outflows and spectra, which can extend up to TeV energies, blazars are laboratories for studying the most extreme physical conditions in any cosmic object. Key questions that can be addressed by Kronos multiwavelength time-series data include:

1. How is a relativistic jet launched and collimated into narrow cones?

2. How is the kinetic energy of a jet converted into ultrarelativistic particles and then to radiation? 
3. What is the underlying cause of the variability and appearance of apparent superluminal knots?

4. How are these sources related to the Galactic "microquasars?"

\subsection{Understanding Jet Physics with Kronos}

The observed jet radiation in blazars is produced by a distribution of relativistic electrons (and possibly positrons) with Lorentz factors as high as $\sim 10^{7}$. The most likely radiative processes include synchrotron emission plus inverse Compton scattering of low-frequency "seed" photons by these electrons. By studying the radiation patterns, we can determine where, and infer how, electrons are accelerated to such high energies. The shapes of the spectra at a given time provide some information, but the crucial test of models is found in spectral variability. In particular, frequency-dependent time delays in the flux variations of the non-thermal continuum are key to understanding the physical processes involved. All monitoring campaigns, to date, have suffered from having too short a duration, limited frequency coverage, or incomplete time coverage that does not allow us to specify correlations and time lags precisely. Even in the best-observed cases ${ }^{6.34 .32 .20}$, relationships among the variations at different wavelengths remain unclear, and, in fact, could be variable ${ }^{39}$ or perhaps even an artifact of Earth occultations ${ }^{\top}$. With its long-period orbit, Kronos is free of such aliasing effects.

\subsection{Origin of the Radiating Electrons and Seed Photons}

In relativistic jet models, the observed radio-to-UV (and X-ray in some BL Lacertae objects) emission is synchrotron radiation, and the X-ray/gamma-ray photons are due to inverse Compton scattering of low-energy "seed photons." How does the jet produce the relativistic electrons responsible for these processes? Are they injected by the central engine at the base of the jet; are they energized by turbulent magnetic fields throughout the jet; or are they accelerated at standing shocks in the radio core or at the fronts of shocks that propagate down the jet, appearing in the radio as superluminal knots? What is the source of seed photons that are scattered to X-ray/gamma-ray energies? These are highly controversial, with solutions ranging from synchrotron photons in the jet (direct or reflected off clouds) to ambient photons from the accretion disk/torus or emission-line clouds.

Each of the above models predicts a particular signature in the multifrequency light curves, so Kronos can distinguish among them definitively, providing critical knowledge on the energetics deep in the heart of the AGN, both near and parsecs away from the central engine. Mapping the jet will determine how and where the ultrarelativistic electrons are produced, characteristics of the radiation environment, and the scale on which most of the energy is dissipated in the jets. This is crucial for interpreting the importance of the gamma-ray emission: do the gamma-rays really dominate the radiative output of a blazar or is this only an illusion of a greater level of relativistic beaming that occurs when the seed photons are external to the jet ${ }^{31.5 .1}$ ?

\section{Stellar-Mass Accretion-Driven Sources}

\subsection{Soft X-Ray Transients}

Soft X-ray transients (SXTs) are interacting binary stars in which a compact star is stripping mass from a companion star. The transferred gas forms an accretion disk as it spirals down to the compact star, viscous stresses in the disk releasing accretion energy. The distinguishing feature of SXTs is X-ray outbursts caused by an increased rate of mass flow through the disk $^{18}$. Outbursts typically last a few months and are separated by periods of quiescence lasting decades or longer. During quiescence the optical light from SXTs is dominated by the companion star and classical techniques can be used to determine the masses and dimensions of the binary system. Because the companion is relatively undistorted by X-ray irradiation, the accuracy of the measurements is much greater than can be achieved for non-transient X-ray binaries ${ }^{4}$; the masses of the component stars have been determined to accuracies better than $10 \%$ in some cases ${ }^{3.12}$. The masses of the compact stars are generally much greater than the upper limit to the mass of a neutron star, $3.2 M_{\odot}$, and, within the context of standard physics, they must, therefore, be black holes. A few SXTs show X-ray bursts whose characteristics imply that they must contain neutron stars. The compact stars in these systems invariably have low masses $\left(\sim 1.4 M_{\odot}\right)$.

SXTs are superb black-hole laboratories. They display most aspects of black-hole accretion, and they allow direct comparisons between accretion onto neutron stars and black holes. For example, SXTs containing black holes emit far less $\mathrm{X}$-ray flux during quiescence than those containing neutron stars" ${ }^{11}$. The extra flux from accreting neutron stars is believed to come from a boundary layer at their surface, a surface absent from accreting black holes. This, in turn, has been hailed as direct evidence for black-hole event horizons, a profound prediction of General Relativity. 
Kronos will give the very first continuous, multiwavelength observations of entire SXT outbursts.

\subsection{Cataclysmic Variables: Accretion in Dwarf Novae}

Cataclysmic variables (CVs) are interacting binary stars in which a white dwarf accretes matter transferred from a normal star $^{37}$. Dwarf novae are a subset of CVs that exhibit repeating outbursts. The outbursts have amplitudes of 2 to 6 magnitudes at optical wavelengths, typically last 3 to 30 days, and recur at irregular intervals of a few weeks to a few months. In these systems, the transferred gas forms a disk as it spirals down to the white dwarf. According to the disk instability model for the outbursts $^{18}$, the viscosity is low between outbursts and the gas accumulates in the disk. When the surface density at some radius reaches a critical threshold, the temperature and viscosity at that radius rise rapidly. This local instability then spreads throughout the disk via a heating wave, producing a large increase in mass flow and energy generation.

Dwarf novae are ideal for investigating the physics of disk accretion. There is much less irradiation and heating than in $\mathrm{X}$ ray binaries or AGN, allowing a cleaner investigation of local physical processes. The rate of mass flow varies greatly, allowing a study of different accretion rates within the same system. Further, there are many bright eclipsing dwarf novae, allowing the disk to be imaged at microarcsecond resolution.

Because the dwarf novae provide such detailed information, we can ask more refined questions about the fundamental physical processes at work: How do spiral waves, magnetic fields, winds, and turbulence transport mass, energy, and angular momentum in disks? What do disks really look like? What is the local spectral energy distribution across the disk, and how is it related to the local mass density and rate of mass flow? What are the properties of the boundary layer between the disk and its central star? What are the energetics and kinematics of the optically thin gas?

The key to answering these questions is to obtain a complete, multiwavelength, time-resolved, observational description of the dwarf nova disk and its variations over the outburst cycle. This has never been achieved. Even the most ambitious campaigns ${ }^{28}$ have been inadequate due to lack of simultaneity and undersampling.

Dwarf nova outbursts seem well explained by thermal/viscous instabilities in the disk. However, the comparisons between observations and theory are primarily qualitative, placing few reliable constraints on the outburst models or the models for viscosity. For example, the evolution of the optical, UV, and X-ray light curves and spectra depends sensitively on the initial location of the instability and on how fast the heating and cooling waves propagate through the disk. However, these structures have never been observed due to a lack of simultaneous multiwavelength observations of sufficient duration. This is the same disk instability thought to apply to SXTs, even though SXTs generate enough radiation to keep the entire disk hot until it nuns out of mass. With data on both SXT and CV outbursts, we can compare the same instability in both strong and weak radiation fields.

Numerical models of CV disks typically produce substructures such as spiral patterns, ellipticity, and the bright spot ${ }^{38.21}$. Observations of these substructures are powerful tests of the calculations and the physics behind them. Continuum structures can be measured by eclipse mapping techniques, and emission-line structures can be measured by both eclipse mapping and Doppler tomography techniques. These structures evolve rapidly over the course of outbursts, requiring observations over several months with time resolution of a few hours.

Kronos is superbly equipped to observe dwarf novae. It will produce nothing less than complete, multiwavelength, timelapse imaging and tomography of dwarf nova disks with both the time resolution and time duration to adequately sample entire outburst cycles. Our basic tools will be multiwavelength spectrophotometry, multiwavelength eclipse mapping, and Doppler tomography of UV and optical emission lines.

Simultaneous, multiwavelength spectrophotometry at X-ray, UV, and optical wavelengths is necessary to deconvolve the contributions of the disk, the boundary layer, and the two stars to the total flux. Eclipse observations yield disk maps with microarcsecond resolutions capable of resolving features as small as spiral patterns and spots. Tomography reveals the kinematics of the gas flows. Together, they yield disk maps with a spectral energy distribution at every point on the disk and boundary layer. The maps will have time resolutions of hours and will cover entire outbursts, yielding true, time-lapse movies of the evolution of accretion disks through the outburst. 


\section{The Kronos Observatory}

\subsection{Science Instruments}

The science instrument package is comprised of a $0.7-\mathrm{m}$ ultraviolet/optical telescope (UVT) and a $0.4-\mathrm{m}$ X-ray telescope (XRT). The telescopes will be co-aligned to within 30 arcsecs. The pointing accuracy of Kronos is determined by the 20 arcsec field-of-view (FOV) of the UVT; the much larger FOV (24 arcmin) of the XRT ensures that a target in the UVT FOV also will be well-centered in the XRT FOV.

In addition to the desired wavelength coverage, the UVT design was driven by the need for high sensitivity and high spectrophotometric precision that would enable the detection and measurement of source fluctuations of less than $1 \%$ on shorter time scales (less than a week or so) and less than $3 \%$ over periods extending up to 300 days. An off-axis parabolic primary mirror brings light to a focus on the Rowland circle of a modified Rowland Mount spectrograph, with mildly toroidal gratings to control astigmatism. The spectrograph features distinct channels for the far ultraviolet $(100-170 \mathrm{~nm}$, resolution $0.15 \mathrm{~nm})$ and the near-UV/optical $(270-540 \mathrm{~nm}$, resolution $0.25 \mathrm{~nm})$.

The XRT incorporates a CCD camera and a conical foil mirror. It is similar to one ASCA SIS module, with a larger mirror aperture, higher angular resolution (resulting in improved sensitivity), an improved $C C D$ camera, a design selected for its simplicity, flight heritage ${ }^{29.30}$, and sensitivity that matches that of the UVT for the key scientific objectives. The XRT mirror is a segmented, thin-foil, conical, grazing-incidence, imaging mirror with a $3.4-\mathrm{m}$ focal length. A $B B X R T$-style thermal precollimator is used to improve the instrument sensitivity below $0.5 \mathrm{keV}$.

\subsection{The Kronos Orbit}

Kronos uses a lunar-assisted open orbit that provides the required long, uninterrupted observing intervals and the low radiation and background environments required to meet the science goals.

Kronos uses phasing loops to achieve a lunar gravitational assist, placing the observatory into a 13.7-day period (one-half the lunar orbit period), inclined $\left(\sim 50^{\circ}\right)$, $\mathrm{HEO}\left(\sim 7 \times 70 R_{\mathrm{E}}\right)$. Phasing loop perigee maneuvers using an on-board propulsion system are used to expand the launch window and to correct for launch vehicle errors ${ }^{22}$. The gravity assist (perilune $\sim 6000 \mathrm{~km}$ ) introduces a $90^{\circ}$ shift between the spacecraft orbit axis and the lunar orbit, ensuring orbit stability over the mission lifetime. A maneuver after the gravity assist establishes the mission orbit. While lunar perturbations cause the perigee, apogee, and inclination to vary between 7 to $18 R_{\mathrm{E}}, 60$ to $70 R_{\mathrm{E}}$, and $25^{\circ}$ to $70^{\circ}$, respectively, simulations have shown that the orbit is stable for at least 6 years, and no orbit maintenance maneuvers are required. The total $\Delta V$ varies, depending on launch date, but analysis yields a value $\Delta V \leq 131 \mathrm{~m} / \mathrm{s}$ for all reasonable cases. Acceptable launch windows occur on roughly half the days of any given month, and there are no constraints on launch year. This family of orbits is relatively new, but the use of phasing loops to target a lunar encounter has precedents on missions such as the Microwave Anisotropy Probe (MAP) and Clementine.

\subsection{The Kronos Mission}

Kronos observations require long pointed observations, often lasting from days to months. In some cases, sampling rates may be a few to several times per day, and in some cases, the sampling will be continuous. Numerical simulations have shown that the science programs will not be adversely affected by occasional gaps in coverage due to source occultations near perigee, time for calibrations, or occasional loss of data. Kronos accommodates several observing modes employed at different stages of the mission:

Long-looks. Some observational programs require both high time resolution and very long duration. Kronos can provide virtually uninterrupted observations for periods up to nearly the 14-day orbital period, and, in some cases, even longer. Longlook observations are used whenever uninterrupted observations are essential.

Multiplexing. Many observations do not require a $100 \%$ duty cycle. Kronos will have a rapid slew capability $(<10$ minutes to slew $180^{\circ}$ ) enabling cyclic observations of several targets. For example, AGNs will usually be observed at intervals of 0.1 to 0.2 days for about 1 hour per visit, so that as many as 3 to 5 objects can be reverberation-mapped contemporaneously.

Target-of-opportunity (TOO). Kronos will be able to respond rapidly to TOOs triggered by observations with other facilities. Turnaround times will generally be less than 24 hours and can be much faster if scheduling blocks are designated for TOO priority. Since TOOs are incompatible with the first two modes above, we will set aside times where TOOs have priority. 
Protocols for interruption of monitoring observations (e.g., the more unique and important a particular event might be, the higher its ranking for displacing ongoing observations) will be established by the Kronos Science Team prior to launch.

\section{ACKNOWLEDGEMENTS}

We are grateful for support of the Kronos science simulations through NASA grant NAG5-8397 to The Ohio State University.

\section{REFERENCES}

1. Blandford, R.D., \& Levinson, A. 1995, ApJ, 441, 79

2. Blandford, R.D., \& McKee, C.F. 1982, ApJ, 255, 419

3. Casares, J., \& Charles, P.A. 1994, MNRAS, 271, L5

4. Charles, P. 1998, in Theory of Black Hole Accretion, ed. M. A. Abramowicz, G. Bjornsson, \& J. E. Pringle (Cambridge: Cambridge University Press), p. 1

5. Dermer, C.D., \& Schlickeiser, R. 1993, ApJ, 416, 458

6. Edelson, R., et al. 1995 ApJ, 438, 120

7. Edelson, R., Griffiths, G., Markowitz, A., Sembay, S., Turner, M.J.L., \& Warwick, R. 2001, ApJ, 554, 274

8. Elvis, M. 2001, ApJ, 545, 63

9. Fabian, A.C., \& Iwasawa, K. 1999, MNRAS, 304, L34

10. Fromerth, M.J., \& Melia, F. 2000, ApJ, 533, 172

11. Garcia, M. R., McClintock, J.E., Narayan, R., Callanan, P., Barret, D., \& Murray, S.S. 2001, ApJ, 553, L47

12. Greene, J., Bailyn, C.D., \& Orosz, J.A. 2001, ApJ, 554, 1290

13. Home, K. 1985, MNRAS, 213, 129

14. Home, K., Peterson, B.M, Collier, S.J., \& Netzer, H. 2002, submitted to PASP (astro-ph/0201182)

15. Kaspi, S., Smith, P.S., Netzer, H., Maoz, D., Jannuzi, B.T., \& Giveon, U. 2000, ApJ, 533, 631

16. Krolik, J.H. 2001, ApJ, 551, 72

17. Laor, A. 1998, ApJL, 505, L83

18. Lasota, J.-P. 2001, New Astronomy Reviews, 45, 449

19. Marsh, T.R., \& Horne, K. 1988, MNRAS, 235, 269

20. Marshall, H.L., Uny, C.M., Sambruna, R.M., \& Pesce, J.E. 2001 ApJ, 549, 938

21. Matsuda, T., Makita, M., and Boffin, H. M. J. 1999, in Disk Instabilities in Close Binary Systems, ed. S. Mineshige \& J.C. Wheeler (Tokyo: Universal Acad. Press), p. 129

22. McGiffin, D., \& Mathews, M. 2000, "Mission Feasibility Study for the Kronos High Earth Orbit," CSC-96-968-19, prepared under the direction of T. Stengle, GSFC, Flight Dynamics Navigation, Attitude, and Information Technology (IDIQ) Task S-32415-G

23. McLure, R.J., \& Jarvis, M.J. 2002, submitted to MNRAS (astro-ph/0204473)

24. Merritt, D., \& Ferrarese, L. 2001, in The Central Kiloparsec of Starbursts and AGN, ed. J. H. Knapen, J. E. Beckman, I. Shlosman, \& T. J. Mahoney, in press (astro-ph/0107134)

25. Peterson, B.M 1993, PASP, 105, 247

26. Peterson, B.M. 1997, An Introduction to Active Galactic Nuclei, (Cambridge: Cambridge University Press)

27. Peterson, B.M. 2001, in The Starburst-AGN Connection, ed. I. Aretxaga, D. Kunth, \& R. Mujica (Singapore: World Scientific), p. 3 (astro-ph/0109495)

28. Pringle, J.E., et al. 1987, MNRAS, 225, 73

29. Serlemitsos, P.J., et al. 1996, ApSS, 239, 177

30. Shibata, R., et al. 1998, Proc. SPIE 3444, 598

31. Sikora, M., Begelman, M.C., \& Rees, M.J. 1994, ApJ, 421, 153

32. Takahashi, T., et al. 2000, ApJ, 542, L105

33. Ulrich, M., Maraschi, L., \& Urry, C.M. 1997, ARAA, 35, 445

34. Urry, C.M., et al. 1997, ApJ, 486, 799

35. Vestergaard, M. 2002, ApJ, 571, 733

36. Wandel, A., Peterson, B.M., \& Malkan, M.A. 1999, ApJ, 526, 579

37. Warner, B. 1995, Cataclysmic Variable Stars, (Cambridge: Cambridge University Press)

38. Whitehurst, R., \& King, A.R. 1991, MNRAS, 249, 25 
39. Zhang, Y. H., Celotti, A., Treves, A., Chiappetti, L., Ghisellini, G., Maraschi, L., Pian, E., Tagliaferri, G., Tavecchio, F., Urry, C.M. 1999 ApJ, 527, 7 